\title{
Effects of Laser Assisted Hatching on Pregnancy Outcomes of Frozen Embryo Transfer Cycles
}

\author{
${ }^{1}$ Dr.Narmadha.R \\ Lecturer in clinical Embryology, \\ Department of Reproductive Medicine and Surgery, \\ Sri Ramachandra Institute of Higher Education And \\ Research, Chennai- India \\ ${ }^{3}$ Dr.Manjula \\ Assistant Professor in clinical Embryology , \\ Department of Reproductive Medicine and Surgery, \\ Sri Ramachandra Institute of Higher Education And \\ Research, Chennai- India
}

\begin{abstract}
\section{> Back Ground:}

The implantation of the embryo into the uterus requires hatching from its zona pellucida $(Z P)$. The inability of the embryo to break its zona pellucida is considered as a factor for implantation failure. Assisted hatching $(\mathrm{AH})$ is performed to make it easier for natural hatching to occur, also providing early embryoendometrium contact, which favors the embryos implantation into the uterus.
\end{abstract}

\section{$>$ Aim}

To evaluate the effect of laser assisted hatching (LAH) on pregnancy rate in frozen embryo transfer cycle.

\section{> Materials \& methods:}

In a prospective observational study a total of 80 patients who underwent frozen embryo transfer(FET) cycles were included in the study. Patients were divided into Laser assisted hatching group (LAH) and no LAH group. In the LAH group, zona thinning was done with the help of laser just prior to the embryo transfer. In the control group no hatching was done before transfer. The main outcome measures were pregnancy rate $\&$ implantation rate.

\section{$>$ Results:}

The baseline characteristics of the 80 patients included in the study ie.,LAH group $(n=40)$ vs no LAH group $(n=40)$, the mean age $(30.85 \pm 5.4$ vs $33 \pm 3.9)$, mean BMI (25.6 \pm 4.1 vs 27.9 \pm 4.5 ), duration of infertility ( $6.18 \pm 4.1$ vs $8.53 \pm 4.7$ ), number of embryos transferred $(\mathbf{2 . 7 5} \pm \mathbf{0 . 8}$ vs $2.7 \pm 0.72)$ respectively. Younger women < 30 years in no LAH group had higher pregnancy rate compared to the LAH group. Between the two groups, patients between 31-35 years in LAH group had higher pregnancy rate compared to no LAH group. The pregnancy rate $(42.85 \%$ vs $54.5 \%)$ in LAH group of women >35years found lower than the no LAH group.

\author{
${ }^{2}$ Dr.N.Sanjeeva Reddy \\ Professor and Head, \\ Department of Reproductive Medicine and Surgery, \\ Sri Ramachandra Institute of Higher Education And \\ Research, Chennai- India \\ ${ }^{4}$ Dr.Sindhuja.N.S \\ Lecturer in clinical Embryology, \\ Department of Reproductive Medicine and Surgery, \\ Sri Ramachandra Institute of Higher Education And \\ Research, Chennai- India
}

\section{Conclusion: \\ LAH seems to be beneficial in women between 31 - 35 years of age group, but LAH does not seem to be beneficial in women > 35 years of age. There is slight increase in pregnancy rates with laser assisted zona thinning in frozen transfer cycle, but it is not statistically significant.}

Keywords:- Frozen Embryo Transfer, Zonapellucida, Laser Assisted Hatching.

\section{INTRODUCTION}

Hatching of the blastocyst is a critical step before implantation into the endometrium of the uterus. Failure to hatch is thought to be one of the factors limiting further embryo development ${ }^{1,2}$. One of the main objectives of Assisted Reproductive Technologies (ART) is improving the embryo implantation rate. ${ }^{3}$

A glycoprotein layer, known as the zona pellucida (ZP), surrounds human embryos and permits only acrosome intact sperm to fertilize the oocyte by blocking the entry of multiple sperm. After fertilization the ZP compresses and shapes the embryo, protecting it from microorganisms and immune cells. At the blastocyst stage, the embryo breaks out of ZP to begin the developmental process; failure at this stage can prevent implantation ${ }^{4}$.

A possible explanation for the low implantation rates is due to observed abnormalities in the hatching in some patients, in advanced maternal age and in frozen-thawed embryo transfer cycles 5 .

Artificially disrupting the $\mathrm{ZP}$ known as assisted hatching $(\mathrm{AH})$ has been proposed as a method to help improve embryo implantation rates in a selected group of patients. A possible benefit of assisted hatching is that it may facilitate an earlier contact between the embryo and the endometrium, allowing for more exposure to important modulators of growth ${ }^{6}$. 
Assisted Hatching using a 1.48- $\mu \mathrm{m}$ diode laser yields a better outcome than $\mathrm{AH}$ using mechanical or chemical methods ${ }^{7}$. Hiraoka et al have shown that vitrification can increase the hardness of zona, and the embryo implantation and clinical pregnancy rates in the half $\mathrm{ZP}$ thinning FET cycle was superior to that of a quarter $\mathrm{ZP}^{8}$.

At present, there are clear benefits of LAH in the subset of Invitro fertilization (IVF) patient population with sub optimal prognosis including advanced reproductive age, repeated implantation failure, poor embryo quality and frozen/thawed embryo transfer cycles.

When compared with those with an intact zona pellucida human embryos that underwent laser-assisted hatching and transferred have increased implantation rates ${ }^{9}$.

Our clinical study focused on the effect of laser zona thinning (laser-assisted hatching; LAH) on the pregnancy outcomes of frozen-thawed ICSI-ET cycles.

\section{METHODOLOGY}

The present study is a prospective observational study was conducted in the Department of Reproductive Medicine and Surgery ,Sri Ramachandra Medical college and Research Institute, porur, Chennai. A total of 80 patients who underwent frozen embryo transfer cycle were included in the study. Patients having $\leq 3$ embryos on day 2 of embryo culture and patients planned for day 2 embryo transfer were excluded from the study.

Patients who underwent frozen transfer were selected in an alternating manner into laser assisted hatching group (LAH study group) and no LAH group (control group). In the LAH group $(\mathrm{N}=40)$, zona thinning was done with the help of laser just prior to the embryo transfer. In the control group $(\mathrm{N}=40)$ no hatching was done before transfer. Institutional Ethical Committee (IEC) approval was obtained to perform the study. Written and informed consent was obtained from the patients participating in the study in the prescribed format approved by IEC.

All Women enrolled in the study underwent controlled ovarian stimulation with antagonist protocol .Semen analysis was performed according to the World Health Organisation criteria (WHO, 2010). Oocyte retrieval was done at $36 \mathrm{hrs}$ after hCG administration under transvaginal ultrasound guidance. Retrieved oocytes with cumulus cells are incubated for 2 hours. Later Oocytes were denuded and their maturity status was assessed. The spermatozoa used for intracytoplasmic sperm injection (ICSI) procedure was prepared by the double density gradient method. Matured oocytes were injected by the prepared sperms. On day 3 of the embryo culture the quality of all embryos were evaluated and graded according to the Istanbul consensus $2011^{10}$. Blastocysts were examined and scored according to Gardner et al. $2000^{11}$.All grade 1 embryos were taken as good quality, grade 2 were taken as fair quality, only grade 3 embryos were taken as poor quality. Embryos were frozen and transfer was planned at a later date.

\section{A. Laser Assisted Hatching}

Laser assisted hatching was performed before Embryo transfer. Day 3 / Day 4/ Day 5 embryos underwent LAH by means of half zona thinning with a non-contact 670nm, 1.48 micrometer infra red diode laser system (Saturn Active 5). Before LAH quality of embryos were evaluated and graded (according to the Istanbul consensus 2011) ${ }^{10}$.Embryos were placed in a culture media droplet over laid with mineral oil in a four well dish above a warm plate and positioned on the inverted microscope stage ,focused with 40x objective with the laser target located on the outer edge of the Zona Pellucida. Thickness of the ZP was measured before LAH. At least 10 , at most 20 shoots for $0.300-0.700 \mathrm{~ms}$ with the size of $10.4 \mu \mathrm{m}-12.0 \mu \mathrm{m}$ was performed to the proper region of $\mathrm{ZP}$ where blastomeres are not adjacent to the inner membrane of embryos. The thickness of zona pellucida was measured after LAH.

\section{B. Vitrification Protocol}

Embryos were frozen on day 3/ day 4 after ICSI by vitrification. Only good quality day 3 embryos were frozen. Good quality embryos were defined as (According to Istanbul consensus 2011). Those having regular blastomeres, $<10 \%$ fragments and no multinucleated blastomeres, containing at least eight cells on day 3/ day4. $15-20 \mu \mathrm{l}$ drops of vial 1(Equilibration solution) and Vial 2 (Vitrification solution) were dispensed into the lid of a $60 \mathrm{~mm}$ dish. Allowing the dish to equilibrate at room temperature $\left(25^{\circ} \mathrm{C} / 37^{\circ} \mathrm{C}\right)$ ensuring that it achieves room temperature (approximately 10 minutes). Embryos were exposed to Vial 1 solution for 5-8 minutes and four drops of Vial 2 solution for 5 seconds each.( Sure life Vitrification medium).The embryos were placed on the cryolock and plunged into liquid nitrogen(-196; C).

\section{Warming Protocol \\ Embryo warming was performed. About $0.2 \mathrm{ml}$ of vial} 1 (1.0 M Sucrose), vial 2 (0.5 M Sucrose), vial 3 (0.25 M Sucrose) and vial 4 (MOPS )were added on the lid of a $60 \mathrm{~mm}$ dish respectively. Allowing the dish to equilibrate at room temperature $\left(25^{\circ} \mathrm{C} / 37^{\circ} \mathrm{C}\right)$ ensuring that it achieves room temperature (approximately 5-10 minutes) Embryos were transferred into solution 1 and left for 3 minutes, solution 2 for 3 minutes and Solution 3 for 3 minutes and transferred them into solution 4 and left for 5 minutes. At the end of 5 minutes embryos were transferred into equilibrated Cleavage medium. Cryopreserved embryos were considered to be survived if $>50 \%$ of the blastomere are intact.

\section{Embryo Transfer}

All the embryos to be transferred to a patient, underwent laser assisted hatching. Embryos were transferred within 20 minutes after hatching. Embryo transfer was performed under trans abdominal ultrasound guidance. 


\section{E. Statistical Analysis}

The collected data was analysed with IBM.SPSS statistics software 16.0 Version. To describe about the data descriptive statistics frequency analysis, percentage analysis was used for categorical variables and for continuous variables the mean and S.D was used. To find the significance difference between the bivariate samples in Independent groups the unpaired sample t-test and analysis of variance test (ANOVA) were used. In all the above statistical tools the probability value .05 was considered as significant level.

\section{OBSERVATION AND RESULTS}

A total of 80 patients who underwent frozen embryo transfer were included in the study, out of which 40 patients grouped under study group (LAH group), 40 patients grouped under control group (No LAH group) respectively. The mean age was significantly different between the two groups and mean body mass index, duration of infertility, number of embryos transferred and survival rate were comparable between both the groups .Ovarian reserve markers such as follicle stimulating hormone(FSH), luteinizing hormone(LH), Estradiol(E2) were comparable between both the groups (Table 1) .
The mean zona pellucida (ZP) thickness before laser assisted thinning was $21.33 \pm 4.8$ and the mean thickness after thinning was $10.9 \pm 3.6$. Majority of the embryos had ZP thickness of $>20 \mu \mathbf{m}$ before LAH and 11-20 $\mu \mathbf{m}$ after LAH in study group.

(Table 2) shows the pregnancy rate when subgroups of $\leq 30$ years , 31-35 years and $>35$-years were compared. Pregnancy rate is higher in study group in $31-35$ years when compared with control group, but did not influence pregnancy rate statistically even though it was clinically significant $(\mathrm{p}=0.534)$.

The statistical analyses, however, show only a trend but the results were not significantly different when we compared the pregnancy rates $(42.5 \%$ vs $52.5 \%)$, implantation rate $(18.1 \%$ vs $28.7 \%)$ and clinical pregnancy rate (30\% vs $45 \%)$ of the study (LAH group) and control group (LAH group) (Table 3 ).

Confounding factors like age, day of transfer ,quality of embryos which can influence pregnancy rate were analysed using logistic regression and was not statistically significant.

\begin{tabular}{|c|c|c|c|}
\hline CHARACTERISTICS & LAH (n=40 ) & NO LAH (n=40) & P value \\
\hline Mean age & $30.8 \pm 5.4$ & $33.0 \pm 3.9$ & 0.048 \\
\hline Mean BMI & $25.6 \pm 4.1$ & $27.9 \pm 4.5$ & 0.48 \\
\hline Duration of infertility & $6.18 \pm 4.1$ & $8.53 \pm 4.9$ & - \\
\hline FSH & $6.9 \pm 2.31$ & $10.87 \pm 18.8$ & 0.190 \\
\hline LH & $5.55 \pm 3.47$ & $6.41 \pm 7.9$ & 0.529 \\
\hline E2 & $56.8 \pm 38.2$ & $49.8 \pm 21.6$ & 0.319 \\
\hline No. of Embryos transferred & $2.75 \pm 0.89$ & $2.7 \pm 0.723$ & 0.785 \\
\hline Survival rate & $85.9 \%$ & $91 \%$ & 0.666 \\
\hline
\end{tabular}

Table 1:- Patient's Baseline Clinical Characteristics In The Laser And No Laser Group.

\begin{tabular}{|c|c|c|c|}
\hline AGE & LAH $(\mathbf{n = 4 0})$ & NO LAH $(\mathbf{n}=\mathbf{4 0})$ & P value \\
\hline$\leq 30$ years & $5 / 17(29.4 \%)$ & $6 / 11(54.5 \%)$ & 0.534 \\
\hline $31-35$ years & $9 / 16(56.2 \%)$ & $9 / 18(50 \%)$ \\
\hline$>35$ years & $3 / 7(42.8 \%)$ & $6 / 11(54.5 \%)$ & \\
\hline
\end{tabular}

Table 2: Pregnancy Rate Related To Age In The Laser And No Laser Group.

\begin{tabular}{|c|c|c|c|}
\hline RESULTS & LAH $(\mathbf{n = 4 0 )}$ & NO LAH (n= 40) & P value \\
\hline Pregnancy rate & $17 / 40(42.5 \%)$ & $21 / 40(52.5 \%)$ & 0.377 \\
\hline Implantation rate & $20 / 110(18.1 \%)$ & $31 / 108(28.7 \%)$ & 0.073 \\
\hline Clinical pregnancy rate & $12(30 \%)$ & $18(45 \%)$ & 0.124 \\
\hline Biochemical & $3(7.55 \%)$ & $1(2.5 \%)$ & 0.311 \\
\hline Miscarriage rate & $2(11.7 \%)$ & $2(9.5 \%)$ & 1.0 \\
\hline
\end{tabular}

Table 3: Pregnancy Outcomes in the Laser and No Laser Groups 


\section{DISCUSSION}

Implantation rate of embryos remains relatively low despite the development in IVF and ICSI and has been indicated that only $15 \%$ of embryos were successfully transplanted into the uterine cavity in the $1990 \mathrm{~s}^{12}$.Selective application of LAH in ART may enhance hatching ability of the embryo. In the process of embryonic development embryo hatching ability plays an important role, the $\mathrm{ZP}$ exhibits good elasticity to become thin with the expansion of the blastocyst that ensures the success of a hatched embryo Thus, a potential mechanism to improve embryo implantation ability may be technological assurance of embryos at an earlier stage of hatching and early contact with the endometrium. The nutrient exchange between the liquid culture and embryos, may produce embryonic development and blastocyst formation which may be accelerated by zona thinning. ${ }^{13,14}$

Age of the female partner plays an important role in infertility which influences the ART procedures outcome. The chance of conception significantly declines over the age of 35 years in women. In our study majority of the patients were in the age group of $<30$ years and was statistically significant this is because of early marriage among Indian women and seek infertility treatment earlier. This can also be due to male factor infertility being the commonest cause. Hence there was early intervention in female partner.

Variation in day of freezing/thawing and the method of cryopreservation may influence clinical outcome. In our study we used vitrification method for freezing of embryos, and included all FET cycles with Day 3, Day 4, and Day-5 embryos, and survival rate was $85.9 \%$ which is less compared to study done by Cai Yun Wan et al ${ }^{15}$. This may be because of variation in the day of freezing/thawing and also quality of the embryos that could affect the outcome.

The mean number of embryos transferred in our study group and no LAH were found to be $(2.75 \pm 0.89$ vs 2.75 \pm 0.89 ).Among 40 patients in study and control group 69 embryos in study group, 77 embryos in control group were in cleavage stage, and 41 embryos in study group, 31 embryos in control group were in blastocyst stage respectively.

In our study $75 \%$ had ZP thickness $>20 \mu \mathrm{m}$ before LAH with mean thickness of $21.33 \pm 4.8$. After LAH $60 \%$ had ZP thickness 11-20 $\mu \mathrm{m}$ with mean thickness of $12.11 \pm 3.4$ respectively. Gabrielsen et al showed assisted hatching may be of benefit for patients having embryos with thick zona pellucida. Thick zona pellucida and/ or decreased variation of the zona pellucida may be associated with advanced female age and poor embryo scores. Also video cinematographic evaluation of zona pellucida thickness variation (ZPTV) correlated strongly with pregnancy $^{16}$.
We studied effect of maternal age on pregnancy rate, even though we found more number of pregnancies in 31 35 age group clinical significance could not be given in our study. Katalin Kanyo et al in his study found that LAH significantly increased the pregnancy rates (18.36\% vs. $11.36 \%$, respectively, $P=0.03$ ) in patients who were over 37 years of age ${ }^{17}$. Whereas, Frydman et al found that LAH did not improve the IVF embryo transfer outcome in woman aged $>37$ years ${ }^{18}$.

Valojerdi et al showed that clinical pregnancy rate was significantly lower with laser assisted zona thinning on vitrified-warmed cleavage stage embryos although it is widely accepted that it gives beneficial results ${ }^{19}$., Whereas in our study only a trend of increased pregnancy rate was found.

There was no statistically significant difference in pregnancy rate between the groups. This has to be confirmed with the large sample size as our sample size is small. Comparing implantation rate in study and control group, it did not show any statistical significance even though there was marginal increase in control group this cannot be given importance as sample size is small. Whereas in the prospective randomized study the implantation rate $(34.2 \%$ versus $23.6 \%, \mathrm{P}=0.021)$ and clinical pregnancy rate $(51.0 \%$ versus $35.3 \%, \mathrm{P}=0.034)$ were significantly higher in the LAH group than the control group $^{15}$. This is probably due to larger sample size and the study design.

In our study, we transferred both good and fair quality embryos in cleavage and blastocyst stage whereas in the study by Shaw-Jackson et al high grade blastocysts were transferred ${ }^{20}$. This was not possible in our study as we had a mixed quality of embryos and this did not influence miscarriage rate.

Eight patients underwent 2 cycles of ET during our study period, they underwent first cycle without LAH and second cycle with LAH .Majority had day 5 transfer and out of which four patients had pregnancy.

There are various confounding factors such as maternal age, day of transfer, quality of embryos which influence pregnancy rate. Logistic regression analysis did not show any significance and did not influence pregnancy rate.

\section{CONCLUSION}

LAH seems to be beneficial in women between 31-35 years of age group, but LAH does not seem to be beneficial in women > 35 years of age. Routine use of laser assisted hatching does not increase pregnancy rate. However, the decision to perform or not to perform LAH on older women should be made on the merits of individual case. LAH may be beneficial in older women with repeated implantation failures. 


\section{REFERENCES}

[1]. Cohen, J., Elsner, C., Kort, H., Malter, H., Massey, J., Mayer, M.P.,Wiemer, K., 1990. Impairment of the hatching process following IVF in the human and improvement of implantation by assisting hatching using micromanipulation. Hum. Reprod. 5, 7-13.

[2]. Cohen, J., 1991. Assisted hatching of human embryos. J. In Vitro Fert.Embryo Transf. 8, 179-190.

[3]. Hammadeh, M.E., Fischer-Hammadeh, C., Ali, K.R., 2011. Assisted hatching in assisted reproduction: a state of the art. J. Assist.Reprod. Genet. 28, 119-128.

[4]. Carroll J, Depypere H, Matthews CD. Freeze-thawinduced changes of the zona pellucida explains decreased rates of fertilization in frozen-thawed mouse oocytes. J Reprod Fertil.1990;90:547-553.

[5]. Cohen, J. (2007). Manipulating embryo development. In Human Preimplantation Embryo Selection (eds K. Elder \& J. Cohen). Abingdon UK: Informa Healthcare, Informa UK.

[6]. J. Cohen, M. Alikani, H.E. Malter, A. Adler, B.E. Talansky, Z. RosenwaksPartial zona dissection or subzonal sperm insemination: microsurgical fertilization alternatives based on evaluation of sperm and embryo morphology FertilSteril, 56 (1991), pp. 696-706

[7]. Martins WP, Rocha IA, Ferriani RA and Nastri CO: Assisted hatching of human embryos: a systematic review and meta-analysis of randomized controlled trials. Hum Reprod Update 17: 438-453, 2011.

[8]. Hiraoka K, Hiraoka K, Horiuchi T, Kusuda T, Okano S, Kinutani M and Kinutani K: Impact of the size of zona pellucida thinning area on vitrified-warmed cleavage-stage embryo transfers: a prospective, randomized study. J Assist Reprod Genet 26: 515-521, 2009.

[9]. Wright, G., S. Wiker, C. Elsner, H. Kort, J. Massey, D. Mitchell, A. Toledo, and J. Cohen. 1990. Observations on the morphology of pronuclei and nucleoli in human zygotes and implications for cryopreservation. Hum.Reprod. 5: 109-115

[10]. Alpha scientists in reproductive medicine and ESHRE special interest group of embryology; The Istanbul consensus workshop on embryo assessment; proceedings of an expert meeting, Human reproduction, volume 26,Issue 6,1 June 2011,pages 1270-1283.

[11]. Classification of blastocyst; Gardner classification

[12]. KutluP,Atvar O and Vanioglu OF: Laser assisted zona thinning technique has no beneficial effect on the ART outcomes of two different maternal age groups.J Assist Reprod Genet 27:457-461,2010.

[13]. Cohen J:Assisted hatching of human embryos.J In vitro fert embryo transfer 8: 179-190, 1991.

[14]. Malter HE and cohen J: Blastocyst formation and hatching in vitro following zona drilling of mouse and human embryos. Gamete Res 24: 67-80,1989.
[15]. Cai-Yun Wan , Cheng Song , Liang-Hui Diao 2014 Laser-assisted hatching improves clinical outcomes of vitrified-warmed blastocysts developed from lowgrade cleavage-stage embryos: a prospective randomized study. Reproductive BioMedicine Online (2014) 28, 582-589.

[16]. Gabrielsen, A,Bhatnagar,P., Petersen,K.and Linderberg,S. (2000) Influence of zona pellucida thickness of human embryos on clinical pregnancy outcome following in vitro ferilisation treatment. J.Assist.Reprod.Genet., 17, 323-328.

[17]. Katalin Kanyo, Jozsef Zeke, Rita Kriston, Zoltan Szücs, Sandor Cseh, Bence Somoskoiand Janos Konc. 2016 The impact of laser-assisted hatching on the outcome of frozen human embryo transfer cycles. Zygote 24 (October), pp. 742-747.

[18]. Frydman, N., Madoux, S., Hesters, L., Duvernoy, C., Feyereisen, E., Le Du, A., Tachdijian, G., Frydman, R. \& Fanchin, R. (2006). A randomized double-blind controlled study on the efficacy of laser zona pellucida thinning on libe birth rates in cases of advanced female age. Hum. Reprod. 21, 2131-5.

[19]. Valojerdi MR, Eftekhari-Yazdi P, Karimian L, Ashtiani SK. Effect of laser zona pellucida opening on clinical outcome of assisted reproduction technology in patients with advanced female age, recurrent implantation failure, or frozen-thawed embryos. Fertil Steril 2008; 90:84 - 91.

[20]. Shaw-Jackson, C., Bertrand, E., Becker, B., Colin, J., Beaudo- in-Chabot, C., Rozenberg, S., Autin, C., 2013. Vitrification of blastocysts derived from fair to poor quality cleavage stage embryos can produce high pregnancy rates after warming. J. Assist. Reprod. Genet. 30, 1035-1042. 